\title{
In-situ TOF neutron diffraction studies of cyclic softening in superelasticity of a
}

\section{NiFeGaCo shape memory alloy}

H. Yang ${ }^{\text {a, b, c }}$, D. Yu ${ }^{\text {b, d }}$, Y. Chen ${ }^{\text {b }}$, J. Mu ${ }^{\text {a }}$, Y. D. Wang ${ }^{\text {a, e, } *, \text { K. An }}{ }^{\text {b, } *}$

${ }^{a}$ Key laboratory for Anisotropy and Texture of Materials, Northeastern University, Shenyang 110819, China

${ }^{b}$ Chemical Engineering Materials Division, Oak Ridge National Laboratory, Oak Ridge, TN 37831, USA

${ }^{c}$ Department of Materials Science and Engineering, the University of Tennessee, Knoxville, TN 37996, USA

${ }^{d}$ School of Chemical Engineering and Technology, Tianjin University, Tianjin 300072, China

${ }^{e}$ State Key Laboratory for Advanced Metals and Materials, University of Science and Technology Beijing, Beijing 100083, China

*Corresponding authors.

E-mail addresses: ydwang@mail.neu.edu.cn (Y. D. Wang), kean@ornl.gov (K. An).

\begin{abstract}
Real-time in-situ neutron diffraction was conducted during uniaxial cycling compression of a $\mathrm{Ni}_{49.3} \mathrm{Fe}_{18} \mathrm{Ga}_{27} \mathrm{Co}_{5.7}$ shape memory alloy to explore the mechanism on its superelasticity at room temperature, which was manifested by the almost recoverable large strains and the apparent cyclic softening. Based on the Rietveld refinements, the real-time evolution of volume fraction of martensite was in-situ monitored, indicating the incremental amount of residual martensite with increasing load cycles. Real-time changes in intensities and lattice strains of $\{h k l\}$ reflections for individual phase were obtained through fitting individual peaks, which reveal the quantitative information on phase transformation kinetics as a
\end{abstract}


function of grain orientation and stress/strain partitioning. Moreover, a large compressive residual stress was evidenced in the parent phase, which should be balanced by the residual martensite after the second unloading cycle. The large compressive residual stress found in the parent austenite phase may account for the cyclic effect on critical stress required for triggering the martensitic transformation in the subsequent loading.

Keywords: Ferromagnetic shape memory alloy; Ni-Fe-Ga-Co; Superelasticity; Neutron diffraction; Stress-induced martensitic transformation.

\section{Introduction}

Ferromagnetic shape memory alloys (FSMAs) such as NiMnGa [1,2], NiFeGa [3,4], NiMnInCo $[5,6]$ have shown great potentials in a variety of applications as actuators, sensors, electromagnetic devices due to the shape memory effect (SME) and superelasticity (SE) under external fields, such as magnetic or stress fields [7,8]. It is well known that both SME and SE are associated with the stress-induced martensitic transformation (SIMT). For SE, the reverse transformation from martensite to parent phase may occur partially or completely after the external stress is released. The SME and SE for FSMAs under mechanical cyclic loading/unloading have been recently investigated extensively. For instance, $\mathrm{Ni}_{49} \mathrm{Mn}_{28} \mathrm{Ga}_{23}$ [001] single crystal possesses $\mathrm{SE}$ above austenite finish temperature $\left(\mathrm{A}_{\mathrm{f}}\right)$ with a recoverable strain of $12 \%$ as evidenced through tensile stress-strain (S-S) measurements [9]. The $\mathrm{Ni}_{54} \mathrm{Fe}_{19} \mathrm{Ga}_{27}$ single crystals orientated with [001] and [123] parallel to the loading direction (LD) demonstrate $\mathrm{SE}$ at $323 \mathrm{~K}$ with reversible compressive strains of up to $3 \%$ under 150 $\mathrm{MPa}$ and $2 \%$ under $300 \mathrm{MPa}$, respectively [10]. A large superelastic strain of $\sim 10 \%$ at $318 \mathrm{~K}$ 
under $100 \mathrm{MPa}$ was also observed in the $\mathrm{Ni}_{49} \mathrm{Fe}_{18} \mathrm{Ga}_{27} \mathrm{Co}_{6}$ single crystal fiber [11]. For $\mathrm{Ni}_{49} \mathrm{Fe}_{18} \mathrm{Ga}_{27} \mathrm{Co}_{6}$ single crystal, SE reaches $\sim 9.5 \%$ at $300 \mathrm{~K}$ under $600 \mathrm{MPa}$ compressive stress [12].

During superelastic deformation of conventional shape memory alloys (SMAs), such as NiTi $[13,14]$, the saturated S-S plateaus are usually observed, which is attributed to that the critical stress required for martensite nucleation is higher than the driving force for growth of martensite variants. Moreover, under the reversible SE, if loaded again, the SMAs show repeatable $S-S$ curves $[15,16]$. In some cases, the SE in FSMAs displays unrecoverable (residual) strain after unloading, and the residual strain increases with repeated loading-unloading sequences accompanied by lower and lower stress plateaus in the S-S curve, i.e. the cyclic softening in SE. For example, CoNiAl polycrystalline FSMAs [17] and the conventional NiTi SMAs [18-20] exhibited the unrecoverable strain and displayed obviously cyclic softening in SE. However, so far the physical mechanism on cyclic loading in SE is still not completely clear. To understand the mechanism on the above-mentioned cyclic softening in SE, the knowledge of phase-specific deformation behavior of the constituent austenite and martensite is thus required during the cyclic loading-unloading. Compared with the traditional post-test measurements, such as SEM and TEM, in-situ neutron diffraction measurements can provide the real-time information on the change in microstructures over a certain sample volume, such as the lattice strain, texture, phase volume fraction, and load sharing for the materials subjected to applied stress [21,22], which is very interesting in exploring the micro-mechanical behavior. This is particularly important for SMAs, whose stress-induced transformation can not be retained after the external stress is 
released.

In this paper, real-time in-situ neutron diffraction was employed to investigate the superelastic cyclic softening behavior of a $\mathrm{Ni}_{49.3} \mathrm{Fe}_{18} \mathrm{Ga}_{27} \mathrm{Co}_{5.7}$ FSMA during uniaxial compression cycles at room temperature. The evolution of phase volume fraction and the strain/stress partitioning for both austenite and martensite during cyclic compressive loadings with increasing maxium stress are revealed. The contributions of phase volume fractions and phase residual stresses to the cyclic softening in SE are elucidated.

\section{Materials and Experiments}

Ingots with a nominal composition of $\mathrm{Ni}_{49.3} \mathrm{Fe}_{18} \mathrm{Ga}_{27} \mathrm{Co}_{5.7}$ (at.\%) were prepared by arc-melting the mixture of the high-purity element metals of $\mathrm{Ni}, \mathrm{Fe}, \mathrm{Ga}$ and $\mathrm{Co}$ in a Ti-getter high-purity argon atmosphere, and further fabricated into rod sucked-cast. The as-cast specimens were homogenized at $1273 \mathrm{~K}$ for $24 \mathrm{hrs}$ in a sealed vacuum silica tube then quenched in water, and finally cut into cylinder samples with $16 \mathrm{~mm}$ in length and $8 \mathrm{~mm}$ in diameter. In-situ time-of-flight (TOF) neutron diffraction experiments were performed with a zero-to-compression cycling mode on the engineering materials diffractometer VULCAN at the Spallation Neutron Source, Oak Ridge National Laboratory (SNS, ORNL). The details of the experimental setup of the in-situ loading experiment can be found in Ref. [23]. Five cycles were completed in the zero-to-compression cycling test with the compression peak stress increasing from $100 \mathrm{MPa}$ in the first cycle to $430 \mathrm{MPa}$ in the last cycle with an interval of $100 \mathrm{MPa}$ over the first four cycles. The sample cracked when the external stress load reached $430 \mathrm{MPa}$ in the fifth cycle, then unloaded. Neutron diffraction data was collected 
continuously while the sample was under external stress. Using the VDRIVE software [24], the continuously recorded neutron data and mechanical data can be synchronized and chopped into 1-min subsets for further Rietveld refinement and single peak fitting analysis. The General Structure Analysis System (GSAS) [25] is used for determining the phase volume fraction by full TOF profile fitting. The structural transformation temperature during cooling/heating was obtained by NOMAD instrument at the SNS, ORNL [26].

\section{Results}

\subsection{Superelasticity characterized by TOF neutron diffraction}

The microstructure of the current alloy before and after deformation was studied by neutron diffraction method. Fig. 1(a) and (b) show the results of full-pattern Rietveld refinement via GSAS of the 1 minute neutron data before loading and at the peak stress of the fifth cycle (430 MPa) respectively. Before loading, the parent austenite phase is $\mathrm{Fm} \overline{3} \mathrm{~m}$ (FCC) structure with a lattice parameter of $\mathrm{a}=\mathrm{b}=\mathrm{c}=5.726 \AA$. When the applied stress reaches 430 $\mathrm{MPa}$, little amount of parent austenite co-exists with the newly-formed martensite phase with the space group of $\mathrm{I} / \mathrm{mmm}$ (BCT), which indicates that the SIMT occurs during the cyclic compressive loadings.
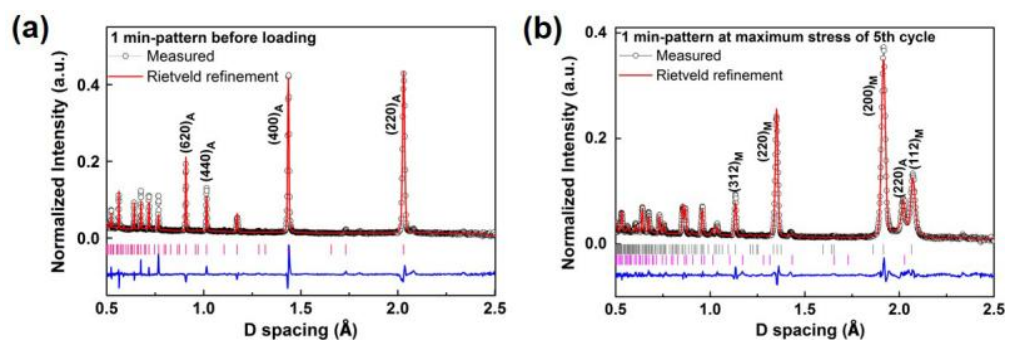

Figure 1. $\mathrm{Ni}_{49.3} \mathrm{Fe}_{18} \mathrm{Ga}_{27} \mathrm{Co}_{5.7}$ alloy neutron diffraction measurements under compressive 
loading: a) neutron diffraction pattern before loading, showing an austenite Fm $\overline{3} \mathrm{~m}$ structure; b) neutron diffraction pattern at the peak stress in the fifth cycle, showing a martensite I4/mmm structure.

Fig. 2(a) shows the macroscopic S-S curves during the room temperature zero-to-compression cycling test. The solid symbols denote the loading values corresponding to the individual neutron diffraction in-situ measurements based on the 1 min-chopping. In the first cycle, the sample behaved elastically without martensitic transformation, since the applied stress was lower than the critical stress to trigger the phase transformation. In the second cycle, the stress plateau appeared, which should correspond to the onset stress $\left(\sigma_{S}\right)$ of martensitic transformation as evidenced from neutron diffraction experiments. The S-S curves of subsequent cycles exhibit a steep slope after the plateau. A kind of stress 'memory' can also be clearly seen from the cyclic loading S-S curves, i.e. the elevated cycle loading stress corresponding to the maximum loading strain in previous cycle coincides with the previous peak stress. Actually, the similar phenomenon on the stress 'memory' has been observed in the NiFeGa single crystal and other alloys [3]. It should be noted that a continuous reduction in $\sigma_{s}$ is observed as seen from Fig. 2(b), featuring cyclic softening in SE. 

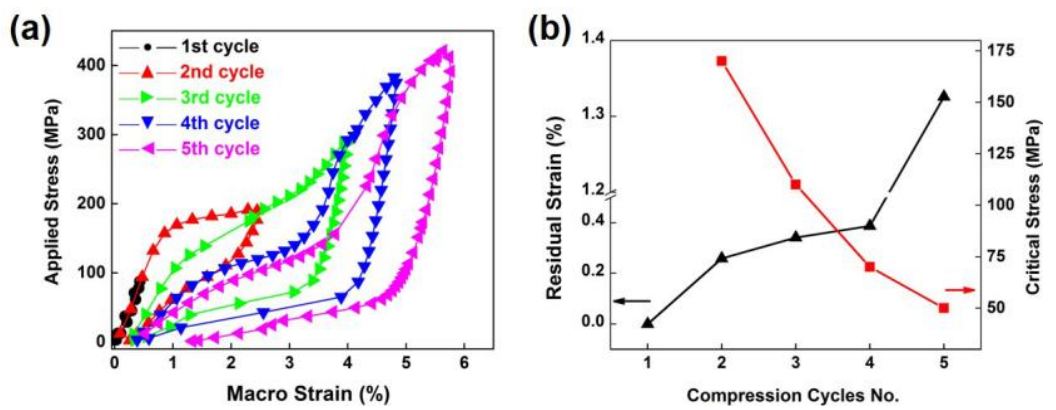

Figure 2. In-situ neutron diffraction measurements under compressive loading: a) cyclic stress-strain behavior of the $\mathrm{Ni}_{49.3} \mathrm{Fe}_{18} \mathrm{Ga}_{27} \mathrm{Co}_{5.7}$ alloy at room temperature; $b$ ) the evolution of residual strain and critical stress by compression cycles.

The unloading stress plateau in the S-S curve is observed due to the reverse martensitic transformation (RMT). It is further found that the onset stress to trigger RMT $\left(\sigma_{r}\right)$ is reduced with increasing loading cycles, which can also be characterized as cyclic softening in SE. The remained macroscopic strain (RMS) after each cycle was extracted from S-S curves, as displayed in Fig. 2(b). There is a slight increase $(<0.4 \%)$ in RMS for the first four cycles, but RMS reaches $\sim 1.3 \%$ in the fifth cycle. Collectively, in the whole cyclic compression process, the studied alloy shows a recoverable maximum strain of $\sim 4.46 \%$ and the apparent softening behavior in SE during reloading.

\subsection{Phase transformation kinetics}

Fig. 3 gives the phase transformation kinetics (PTK) curve of martensite at different loading cycles as a function of loading strain. There are two regions in PTK, i.e. the rapid growth stage of martensite and the saturation stage of martensite. The martensite grows rapidly while the external stress reaches $\sigma_{s}$. After the volume fraction of martensite 
approaches $92 \%$, the martensite increases slightly in the saturation stage while the macroscopic strain still increases from $\sim 3.8 \%$ to $5.8 \%$. Residual martensite can still be observed after complete unloading, which is shown in the inset of Fig. 3 with the volume fraction of $4 \%, 13 \%, 35 \%$ and $55 \%$ in the second, third, fourth and fifth cycle, respectively. The above findings indicate that the evolution of volume fraction of residual martensite correlates well with the incremental change in RMS for elevated loading cycles.

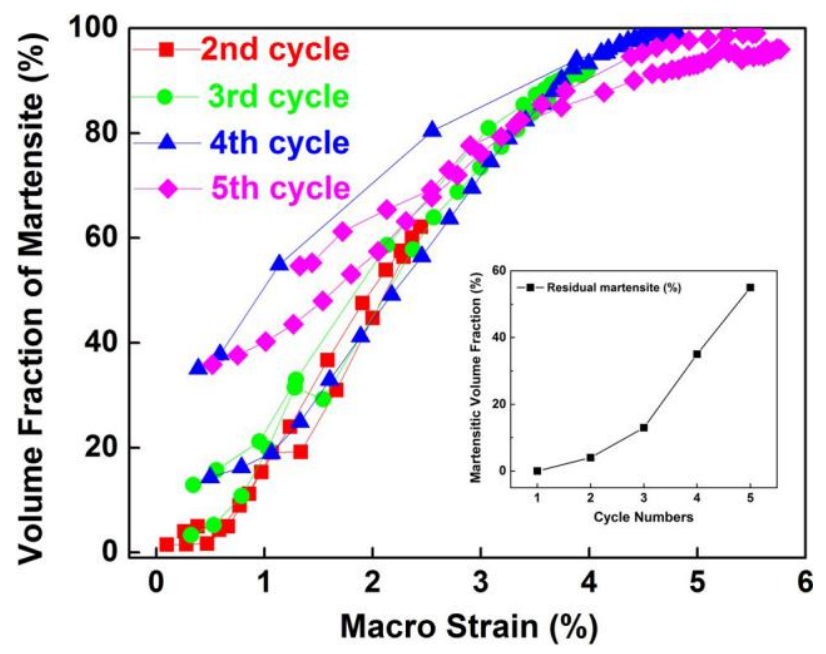

Figure 3. The evolution of the volume fraction of martensite during cyclic compressive loadings. The inset is the residual volume fraction of martensite after each cycle unloading.

The evolution of normalized diffraction intensity is related to their grain/variant-preferred transformation selections for austenite and martensite. According to the typical phase transformation crystallography relationship between parent austenite and martensite phases [27], i.e. $(100)_{A} / /(110)_{M}$ and $(110)_{A} / /(100)_{M}$, the integral intensity for martensite $(220)_{M}$ reflection increases under all loading cycles, accompanied by the decrease in $(400)_{\mathrm{A}}$ diffraction intensity for austenite (Fig. 4). It should be noted that, after 
unloading, the intensity of $(112)_{\mathrm{M}}$ and $(200)_{\mathrm{M}}$ for martensite does not diminish but accumulates progressively with cyclic loading.
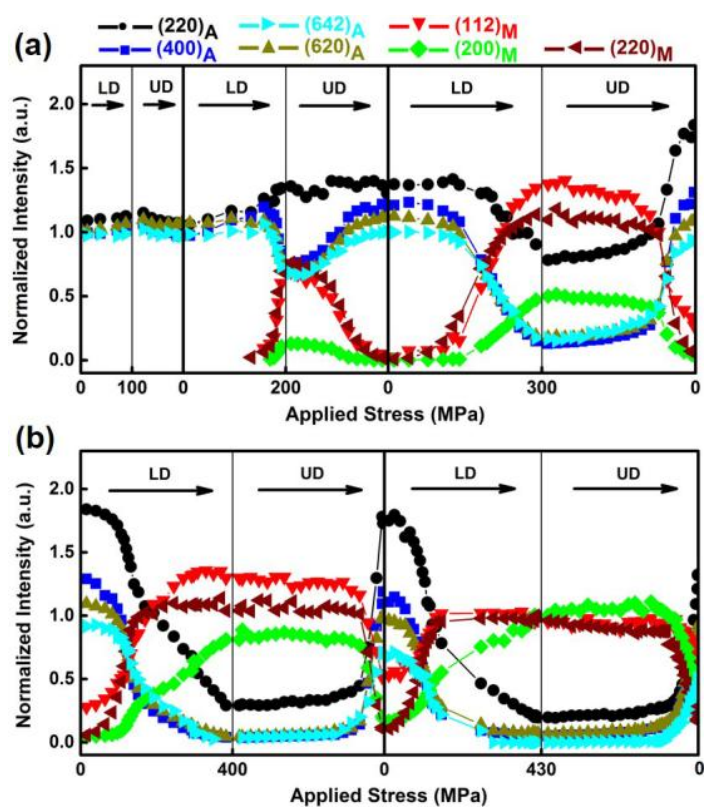

Figure 4. Evolution of normalized intensity of austenite and martensite reflections with applied stress: a) the first cycle to third cycle; b) the fourth cycle to fifth cycle. The normalized intensity of each austenite reflection is based on the initial intensity before loading, while that of each martensite reflection is based on the intensity at the peak stress in the fifth cycle.

It is interesting to note that the intensity for the austenite $(220)_{\mathrm{A}}$ reflection shows a significant increase, i.e. about 1.5 times higher than its initial value after complete unloading. Correspondingly, the intensity for the $(642)_{\text {A }}$ reflection shows an obvious decrease, i.e. about 0.6 times lower than the initial value. The significant change in diffraction intensities for $(220)_{\mathrm{A}}$ and $(642)_{\mathrm{A}}$ is probably due to that the formation of deformation twinning from parent phase [642] parallel to LD prefers to transform into the grains orientated with [220] parallel 
to LD during cyclic loadings. It indicates the austenite cannot completely revert to the initial state via the deformation twinning process after external unloading. Moreover, this also denotes that the accumulated residual strain observed after unloading is closely related to the deformation twinning of parent austenite phase [28].

\subsection{Micro-mechanical behavior during compressive loading cycles}

Based on the above-mentioned analysis, it is deduced that the instability of $\sigma_{s}$ and accumulation of residual strain in the studied $\mathrm{Ni}_{49.3} \mathrm{Fe}_{18} \mathrm{Ga}_{27} \mathrm{Co}_{5.7}$ alloy is mainly due to the gradual redistribution of the internal stress caused by the motion of twin boundaries, i.e., incomplete detwinning of martensite variants. This can be evidenced from the evolution of austenite and martensite lattice strains during the whole loading cycles, as shown in Fig. 5.
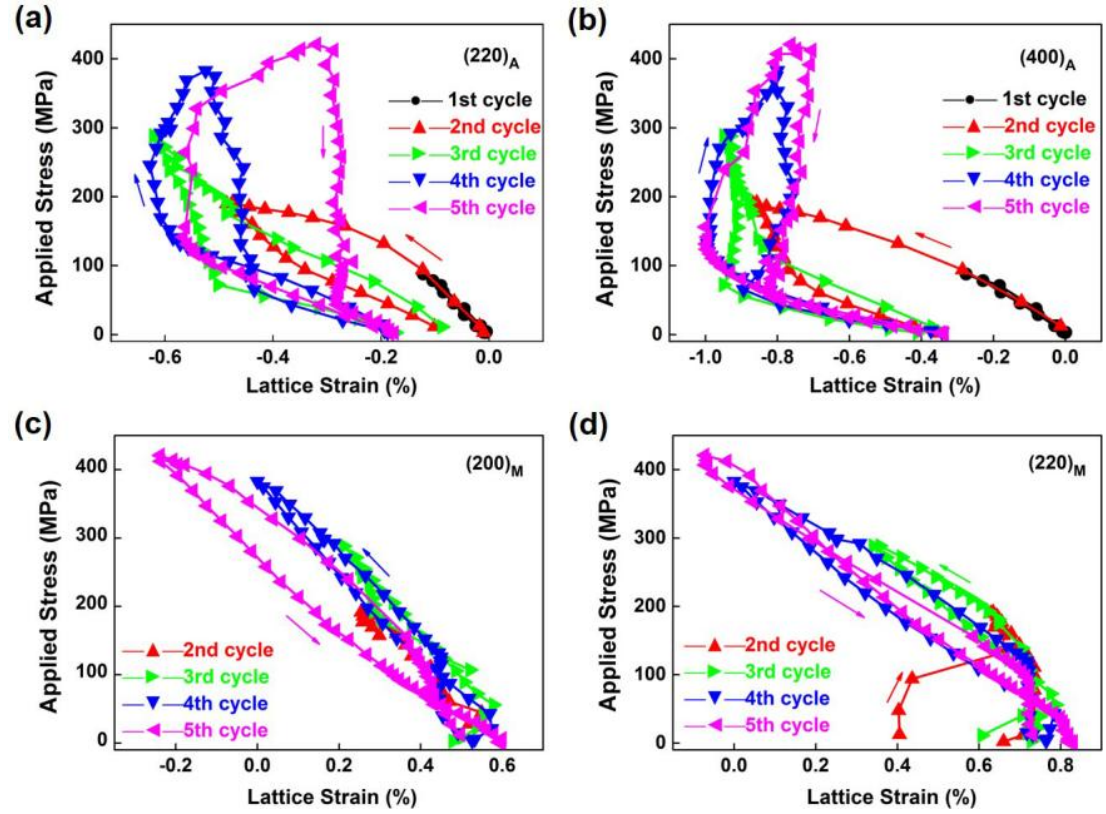

Figure 5. The lattice strain evolution with compressive stress during the cyclic compression: a) austenite (220) plane; b) austenite (400) plane; c) martensite (200) plane; d) martensite (220) 
plane. Each cycle is shown in different color. Since the stress free parameters of martensite reflections are not existence in the beginning, relative values of lattice strain normalized with respect to the corresponding values of martensite reflections recorded at the fourth cycle peak stress are calculated and plot in the figure.

The lattice strain during loading, $\epsilon_{h k l}$, can be calculated from the shift of a diffraction peak position as following Eq. (1):

$$
\epsilon_{h k l}=\frac{d_{h k l}-d_{h k l, 0}}{d_{h k l, 0}}
$$

where the $d_{h k l}$ is the d-spacing determined from the $h k l$ reflection for a given load, and $d_{h k l, 0}$ refers to the value for zero load, a reference state of the experiment. For the austenite phase, the $d_{h k l}$ before loading can be used as the stress-free reference. However, this method is not practical for the martensite because they nucleate under stress, which start to carry loads at their appearance. One illustration option that related literature uses is to normalize the lattice strain for martensite the corresponding maximum strain value in the cycle [15]. Thus, we calculate the strain with reference to the initial unloading part of the fourth cycle with most martensitic phase to demonstrate the straining behavior (excluding the fifth cycle due to cracks during the loading).

The change of lattice strain reflects the phase stresses evolution and the load sharing of each phase. The martensite phase denotes an approximate linear behavior corresponding to the compressive loadings as shown in Fig. 5(c) and (d) which is different from the parent phase. The initial non zero strains of the martensite $\left(0.25 \%\right.$ for $(200)_{M}$ and $0.37 \%$ for $\left.(220)_{M}\right)$ exist at the beginning of second cycle, which indicates the martensite nucleates under 
compressive state. Subsequently, the stress-induced martensite reverts to a certain lattice strain state rapidly after unloading completely in each cycle. The martensite phase seems to exhibit almost elastic deformation under the present mechanical loading, thus implying that the large macroscopic strain variation could be mainly caused by the martensitic generation and grain reorientation.

The lattice strain of austenite grains cannot revert to initial state after cyclic deformation. For example, the lattice strain increase rate of austenite (220) (Fig. 5(a)) reduces since the SIMT occurs in second cycle during compressive loading process, and this rate reduction behavior in third cycle becomes more profound. The lattice strain of austenite decreases when the strain beyond $\sim 0.65 \%$ in fourth cycle, further, the reduction becomes more apparent along with the trend of the former cycle in fifth cycle. Combining with the volume fraction of martensite, it indicates that austenite left little in bulk and martensite sustains most of the external stress since fourth cycle, which explains the lattice strain relaxation behavior in austenite phase during compressive loadings. In the unloading process of austenite (220), the residual strain exists since second cycle, demonstrating unequivocally that the permanent residual stress may be due to the martensitic generation.

\section{Discussion}

In order to understand the softening behavior in SE during compressive loadings, it is necessary to study the stress partition in the cyclic compressive deformation. For an elastic isotropy material, the component stress $\left(\sigma_{h k l}\right)$ should be consistent with the macro-stress stress $\left(\sigma_{G}\right)$. Considering the strong elastic anisotropy in FSMAs [15,27], which has been 
evidenced from $h k l$-grains lattice strain in the studied material obviously, the relationship between the component stress $\sigma_{h k l}$ and macro-stress $\sigma_{G}$ is not consistent anymore. In elastic deformation stage, the applied stress would be redistributed into each oriented $h k l$-grains due to the elastic anisotropy. When the SIMT occurs, the load sharing of specific $h k l$-grains would deviate from the macro-stress because of the anisotropy and SIMT in the material.

As shown in Fig. 5, upon unloading process, lattice strain of specific hkl-grain in parent phase is not fully reverted and introduces the $h k l$-grain residual stress, $\sigma^{r e s}$. Generally, the force to trigger the specific $h k l$-grain martensitic transformation is constant. It can be obtained as fellows [27],

$$
\sigma_{h k l}=\sigma_{G}+\sigma^{r e s}
$$

where the $\sigma_{h k l}$ is component stress, $\sigma_{G}$ is macro-stress to trigger the martensitic transformation, and $\sigma^{\text {res }}$ is $h k l$-grain residual stress. It seems reasonable that cyclic softening in SE is caused by $\sigma^{r e s}$. When the SIMT occurs at second compressive loading and following cycles, the $\sigma^{\text {res }}$ increases gradually on account of the specific $h k l$-grains residual strain accumulates in material (Fig. 2(b)). In order to keep the constant of $\sigma_{h k l}$, the $\sigma_{G}$ becomes lower by elevated cycles which is corresponding with the increase of the $\sigma^{\text {res }}$. Therefore, it may be speculated that the occurrence of residual stress may reduce the critical stress to trigger the martensitic transformation and behaves the cyclic softening.

\section{Conclusion}

In this paper, real-time in-situ neutron diffraction was used to reveal the mechanism on $\mathrm{SE}$ and the effect of cyclic loading on SE softening in the $\mathrm{Ni}_{49.3} \mathrm{Fe}_{18} \mathrm{Ga}_{27} \mathrm{Co}_{5.7}$ FSMA under 
compression mode at room temperature. Based on the results of Rietveld refinement and single peak fitting, the in-situ TOF experiments provide rich information on the evolution of integral intensities, elastic lattice strains and volume fraction of martensite. The SE was attributed to the reversible SIMT during compression loading/unloading cycles. It is verified in our experiments that accumulated residual strain after unloading is accompanied with the cyclic softening behavior. Our experiments also confirm that parent austenite phase cannot completely revert to the initial state via the deformation twinning during cyclic compressive loading process. Moreover, it is concluded that the residual stress, not only lowers the critical applied stress to initiate the martensitic transformation, but retards the reverse martensitic transformation, leading to the residual martensite as well as some residual macroscopic strains.

\section{Acknowledgements}

H. Y. and Y. D. W. would like to thank the funding support by by the National Science Foundation of China (NSFC) (Grant No.s 51471032 and 51527801). H. Y. would like to thank the funding support by the National Basic Research Program of China (973 Program) under Contract No. 2012CB619405 and the China Scholarship Council for the financial support during the visit to University of Tennessee, TN, SNS, ORNL. Neutron scattering experiment was carried out at Spallation Neutron Source (SNS) which is national user facilities sponsored by the Scientific User Facilities Division, Office of Basic Energy Sciences (BES), U.S. Department of Energy. 


\section{References}

[1] M. F. Qian, X. X. Zhang, L. S. Wei, L. Geng, H. X. Peng, Effect of chemical ordering annealing on martensitic transformation and superelasticity in polycrystalline Ni-Mn-Ga microwires, J. Alloy. Compd. 645 (2015) 335-343.

[2] Y. D. Wang, Y. Ren, H. Q. Li, H. Choo, M. L. Benson, D. W. Brown, P. K. Liaw, L. Zuo, G. Wang, D. E. Brown, E. E. Alp, Tracing memory in polycrystalline ferromagnetic shape memory alloys, Adv. Mater. 18 (2006) 2392-2396.

[3] N. Ozdemir, I. Karaman, N. A. Mara, Y. I. Chumlyakov, H. E. Karaca, Size effects in the superelastic response of $\mathrm{Ni}_{54} \mathrm{Fe}_{19} \mathrm{Ga}_{27}$ shape memory alloy pillars with a two stage martensitic transformation, Acta Mater. 60 (2012) 5670-5685.

[4] Z. H. Liu, G. H. Wu, Y. Liu, Stress-induced martensitic transformation of a $\mathrm{Ni}_{54} \mathrm{Fe}_{19} \mathrm{Ga}_{27}$ single crystal in compression, Intermetallics 14 (2006) 1493-1500.

[5] V. Zhukova, M. Ipatov, A. Granovsky, A. Zhukov, Magnetic properties of Ni-Mn-In-Co Heusler-type glass-coated microwires, J. Appl. Phys. 115, (2014) 17A939.

[6] H. B. Huang, X. Q. Ma, J. J. Wang, Z. H. Liu, W. Q. He, L. Q. Chen, A phase-field model of phase transitions and domain structures of NiCoMnIn metamagnetic alloys, Acta Mater. 83 (2015) 333-340.

[7] A. S. Turabi, H. E. Karaca, H. Tobe, B. Basaran, Y. Aydogdu, Y. I. Chumlyakov, Shape memory effect and superelasticity of NiMnCoIn metamagnetic shape memory alloys under high magnetic field, Script. Mater. 111 (2016) 110-113.

[8] M. Chmielus, X. X. Zhang, C. Witherspoon, D. C. Dunand, P. Müllner, Giant magnetic-field-induced strains in polycrystalline Ni-Mn-Ga foams, Nat. Mater. 8 (2009) 863.

[9] V. A. Chernenko, E. Villa, D. Salazar, J. M. Barandiaran, Large tensile superelasticity from intermartensitic transformations in Ni49Mn28Ga23 single crystal, Appl. Phys. Lett. 108 (2016) 071903.

[10]R. F. Hamilton, C. Efstathiou, H. Sehitoglu, Y. Chumlyakov, Thermal and stress-induced martensitic transformations in $\mathrm{NiFeGa}$ single crystals under tension 
and compression, Script. Mater. 54 (2006) 465-469.

[11]K. Oikawa, R. Saito, K. Anzai, H. Ishikawa, Y. Sutou, T. Omori, A. Yoshikawa, V. A. Chernenko, S. Besseghini, A. Gambardella, R. Kainuma, K. Ishida, Elastic and superelastic properties of $\mathrm{NiFeCoGa}$ fibers grown by micro-pulling-down method, Mater. Trans. 50 (2009) 934-937.

[12]E. Panchenko, Y. Chumlyakov, H. J. Maier, E. Timofeeva, I. Karaman, Tension/compression asymmetry of functional properties in [001]-oriented ferromagnetic NiFeGaCo single crystals, Intermetallics 18 (2010) 2458-2463.

[13]J. M. McNaney, V. Imbeni, Y. Jung, P. Papadopoulos, R. O. Ritchie, An experimental study of the superelastic effect in a shape-memory Nitinol alloy under biaxial loading, Mech. Mater. 35 (2003) 969-986.

[14]C. Elibol, M. F. X. Wagner, Strain rate effects on the localization of the stress-induced martensitic transformation in pseudoelastic NiTi under uniaxial tension, compression and compression-shear, Mater. Sci. Eng. A 643 (2015) 194-202.

[15]P. Sedmák, P. Šittner, J. Pilch, C. Curfs, Instability of cyclic superelastic deformation of NiTi investigated by synchrotron X-ray diffraction, Acta Mater. 94 (2015) 257-270.

[16]A. Ahadi, Q. Sun, Effects of grain size on the rate-dependent thermomechanical responses of nanostructured superelastic NiTi, Acta Mater. 76 (2014) 186-197.

[17]C. Efstathiou, H. Sehitoglu, A. J. Wagoner Johnson, R. F. Hamilton, H. J. Maier, Y. Chumlyakov, Large reduction in critical stress in Co-Ni-Al upon repeated transformation, Script. Mater. 51 (2004) 979-985.

[18]Q. Kan, C. Yu, G. Kang, J. Li, W. Yan, Experimental observations on rate-dependent cyclic deformation of super-elastic NiTi shape memory alloy, Mech. Mater. 97 (2016) $48-58$.

[19]X. Xie, Q. Kan, G. Kang, J. Li, B. Qiu, C. Yu, Observation on the transformation domains of super-elastic NiTi shape memory alloy and their evolutions during cyclic loading, Smart Mater. Struct. 25 (2016) 045003.

[20]A. Sibirev, S. Belyaev, N. Resnina, Softening process during reverse martensitic transformation in NiTi shape memory alloy, J. Alloy. Compd. 661 (2016) 155-160. 
[21]H. Yang, Y. Chen, H. Bei, C. R. dela Cruz, Y. D. Wang, K. An, Annealing effects on the structural and magnetic properties of off-stoichiometric Fe-Mn-Ga ferromagnetic shape memory alloys, Mater. Des. 104 (2016) 327-332.

[22]D. Yu, K. An, Y. Chen, X. Chen, Revealing the cyclic hardening mechanism of an austenitic stainless steel by real-time in situ neutron diffraction, Script. Mater. 89 (2014) 45-48.

[23]K. An, H. D. Skorpenske, A. D. Stoica, D. Ma, X. L. Wang, E. Cakmak, First in situ lattice strains measurements under load at VULCAN, Metall. Mater. Trans. A 42 (2011) 95 .

[24]K. An, X. L. Wang, A. D. Stoica: Vulcan Data Reduction and Interactive Visualization Software (VDRIVE), http://www.ornl.gov/ k6e/VDRIVE/, (2010).

[25]B. H. Toby, EXPGUI, a graphical user interface for GSAS, J. Appl. Cryst. 34 (2001) 210.

[26]J. Neuefeind, M. Feygenson, J. Carruth, R. Hoffmann, K. Chipley, The nanoscale ordered Materials diffractometer NOMAD at the Spallation Neutron Source SNS, Nucl. Instrum. Methods Phys. Res. Sect. B: Beam Interact. Mater. 287, 68-75 (2012).

[27]P. Šittner, P. Lukáš, D. Neov, M. R. Daymond, V. Novák, G. M. Swallowe, Stress-induced martensitic transformation in $\mathrm{Cu}-\mathrm{Al}-\mathrm{Zn}-\mathrm{Mn}$ polycrystal investigated by two in-situ neutron diffraction techniques, Metall. Mater. Trans. A 324 (2002) 225-234.

[28]K. Gall, H. J. Maier, Cyclic deformation mechanisms in precipitated NiTi shape memory alloys, Acta Mater. 50 (2002) 4643-4657. 